\title{
Development of a Two-Temperature Open-Source CFD Model for Hypersonic Reacting Flows
}

\author{
Vincent Casseau*, Thomas J. Scanlon ${ }^{\dagger}$ and Richard E. Brown ${ }^{\ddagger}$ \\ University of Strathclyde, Glasgow, G1 1XJ, UK
}

The highly complex flow physics that characterise re-entry conditions have to be reproduced by means of numerical simulations with both an acceptable level of accuracy and within reasonable timescales. In this respect, a new CFD solver, hyFoam, has been developed within the framework of the open-source CFD platform OpenFOAM for modelling hypersonic reacting flows. hyFoam has been successfully validated for two 0-degree adiabatic heat bath test cases and the limitations of a one-temperature CFD model have been highlighted. To cope with high-temperature gas chemistry, the internal energy has been decomposed into its elementary energy modes, thus introducing the translational-rotational and the vibrational temperatures. A two-temperature CFD model is being implemented in order to attain a better agreement between CFD and DSMC results. Validation of the code for a single species has been executed while mixture-related libraries are currently being developed. The vibrational-translational relaxation time formulation has also been presented and discussed.

\section{Nomenclature}

$\begin{array}{llll}\text { Symbols } & & h & \text { enthalpy per unit mass of species } \\ \beta & \text { temperature exponent in the Arrhenius law } & h^{\circ} & \text { chemical enthalpy } \\ \delta & \text { Kronecker delta } & \mathcal{J} & \text { species diffusion vector } \\ \zeta & \text { degree of freedom } & k_{f} & \text { forward reaction rate constant } \\ \theta_{v} & \text { characteristic vibrational temperature } & \mathcal{M}_{i} & \text { molecular weight of species } i \\ \kappa & \text { thermal conductivity } & \mathcal{M}_{j k} & \text { reduced mass of species } j \text { and } k \\ \lambda & \text { bulk viscosity } & n & \text { number density } \\ \Lambda & \text { series of coefficients } & p & \text { pressure } \\ \mu & \text { shear viscosity } & \mathbf{q} & \text { heat conduction vector } \\ \rho & \text { gas density } & Q_{V-\mathrm{ch}} & \text { vibration-chemistry coupling source term } \\ \rho_{k} & \text { partial density of species } k & Q_{V-T} & \text { vibrational-translational energy transfer } \\ \sigma_{v} & \text { limited collision cross-section } & Q_{V-V} & \text { vibrational-vibrational energy transfer } \\ \boldsymbol{\tau} & \text { shear stress tensor } & R & \text { specific gas constant } \\ \tau_{V-T} & \text { vibrational-translational relaxation time } & s & \text { number of species in the mixture } \\ \dot{\omega} & \text { net mass production of species } & T & \text { temperature or overall temperature } \\ & & T_{a} & \text { temperature of activation } \\ A & \text { pre-exponential factor in the Arrhenius law } & T_{t, r, v} & \text { molecular temperatures } \\ \bar{c} & \text { average molecular speed } & u, v, w & \text { components of the velocity vector } \\ C & \text { species concentration } & \mathcal{U} & \text { vector of conserved quatities } \\ e & \text { energy per unit mass of species } & \mathbf{v} & \text { diffusion velocity vector } \\ E & \text { total energy } & \mathcal{W} & \text { source vector } \\ E_{t, r, v} & \text { energy associated with one energy mode } & X & \text { species molar fraction } \\ \mathcal{F} & \text { flux vectors } & Z_{r} & \text { rotational collision number }\end{array}$

${ }^{*}$ PhD Researcher, Department of Mechanical and Aerospace Engineering, Student Member AIAA.

${ }^{\dagger}$ Senior Lecturer, Department of Mechanical and Aerospace Engineering.

¥Professor and Director of the Centre for Future Air-Space Transportation Technology (cFASTT), Department of Mechanical and Aerospace Engineering, Senior Member AIAA. 
Subscripts and superscripts

$\begin{array}{llll}\text { int } & \text { internal } & t r & \text { translational-rotational } \\ r & \text { rotational } & v & \text { vibrational } \\ t & \text { translational } & 0 & \text { initial }\end{array}$

\section{Introduction}

$\mathrm{H}^{\mathrm{i}}$ GH-SPEED vehicles flying at altitudes around ten times higher than today's aircraft represent a future vision of air-space transportation. From an engineering, environmental and societal viewpoint, considerable obstacles remain to be resolved to make this future a reality. ${ }^{1}$ One of the barriers that needs to be overcome concerns the thorough understanding and characterisation of the aerothermodynamic flow conditions experienced by the vehicle as it enters the Earth's atmosphere. ${ }^{2}$ This point is of crucial importance to preserve the integrity of the aircraft in the searing conditions of the descent phase.

The highly complex flow physics that characterise re-entry conditions have to be reproduced by means of numerical simulations with both a good level of accuracy and within reasonable timescales. Hypersonic re-entry vehicle flow fields at intermediate altitudes - ranging from 60 to $80 \mathrm{~km}$ - contain a mixture of continuum and non-continuum (rarefied) regions. Navier-Stokes solutions using conventional computational fluid dynamics (CFD) are appropriate for continuum flows but fail to accurately predict non-continuum flow behaviour. Conversely, the direct simulation Monte Carlo (DSMC) particle-based methodology is the preeminent technique for rarefied flows but requires significant computational effort when solving continuum regions. The craft may also encounter a chemically reacting environment that can have a significant influence on aerodynamic performance and surface heating. Numerical models that fail to incorporate such reacting flows miss an essential part of the flow physics surrounding the vehicle.

The need for reliable numerical tools that can capture thermochemical non-equilibrium with high-fidelity has gained momentum recently, principally due to NASA's upcoming critical missions involving the next Crew Exploration Vehicle, Orion, ${ }^{3}$ and complex systems to allow high-mass payload entries in the Martian atmosphere. ${ }^{2}$ Among the most popular CFD codes dedicated to the study of the hypersonic regime are NASA's DPLR ${ }^{4}$ (Data-Parallel Line Relaxation) and LAURA ${ }^{5}$ (Langley Aerothermodynamic Upwind Relaxation Algorithm), VULCAN ${ }^{6}$ (Viscous Upwind aLgorithm for Complex flow ANalysis), US3D ${ }^{7,8}$ (UnStructured 3D) of the University of Minnesota, and LeMans ${ }^{9,10}$ (the Michigan Aerothermal Navier-Stokes Solver) of the University of Michigan. They have all adopted a similar strategy to cope with thermochemical non-equilibrium where the gas mixture is no longer described by a single temperature but by at least two distinct molecular temperatures. ${ }^{11}$ These temperatures are derived from the decomposition of the internal energy into its elementary energy modes thus distinguishing the translational-rotational temperature from its vibrational counterpart. Each of the CFD codes described above contain differences which are mainly due to the distinct representation of energy exchange between the energy modes. ${ }^{3}$

In order to address the issues described above a new CFD solver has been developed within the framework of the open-source CFD platform OpenFOAM ${ }^{12}$ for modelling hypersonic reacting flows. Contributions of competing mechanisms are considered in isolation by focusing on a 0 -degree adiabatic heat bath and by validating the chemistry module independently from the two-temperature model. Lack of experimental or flight data and uncertainties in the determination of reaction rates and relaxation times force us to reflect upon any comparisons between the CFD and DSMC results, DSMC being often regarded as an experimental analogue. Hence, the DSMC solver, $d s m c F o a m$, developed and validated ${ }^{13,14}$ at the University of Strathclyde, will serve as a benchmark tool for the validation of our CFD code. The code possesses new features such as the ability to model the vibrational internal energy mode and adopts the Quantum Kinetic approach, recently introduced by Bird, for chemical reactions. ${ }^{15}$ Post-collision energy redistribution between the internal energy modes is executed using the Larsen-Borgnakke procedure. ${ }^{13,16}$ 


\section{Methodology}

The work that has been carried out within OpenFOAM can be split into two main sections. The first consisted of improving and validating the current chemistry module using a standard one-temperature model. This then set the basis for a two-temperature CFD arrangement. These are the two stages that are presented in the following sub-sections. Ultimately, the chemistry part and the new thermodynamic libraries will be reunited to form a two-temperature solver for simulating high-temperature reacting mixtures.

\section{A. Chemistry solver}

A suitable OpenFOAM CFD solver, rhoCentralFoam, has first been identified and assessed. This solver has been created to resolve high-speed compressible flows ${ }^{17}$ making use of central-upwind interpolation schemes of Kurganov and Tadmor. ${ }^{18}$ It has produced comparable results to those given by the MISTRAL flow solver, ${ }^{19}$ for continuum hypersonic simulations over a hollow cylinder. ${ }^{20}$ Basic chemistry features have been added to rhoCentralFoam by incorporating parts of the solver reactingFoam, an OpenFOAM solver dealing with combustion. This enables us to include chemical reactions along with their type and rates and to introduce quantities related to the species mixture, such as mass fraction or number density. The newly blended code has been given the name hyFoam. The solution process then consists of resolving a set of ordinary differential equations (ODE) and species mass fractions. Validation of the new code implementation has been carried out by initially considering the reversible reaction of hydrogen and iodine to produce hydrogen-iodide in the reaction $\mathrm{I}_{2}+\mathrm{H}_{2} \rightleftharpoons 2 \mathrm{HI}$. This chemical reaction occurs at constant temperature and an analytical solution of the species concentration over time can be easily derived. ${ }^{21}$ The forward and reverse rate constants at $T=700 \mathrm{~K}$ are also to be found in the aforementioned reference. Species concentrations plotted with respect to time for both the analytical solution and hyFoam are shown in Figure 1. The results demonstrate a highly satisfactory agreement thus validating this first stage of the new hyFoam solver implementation.

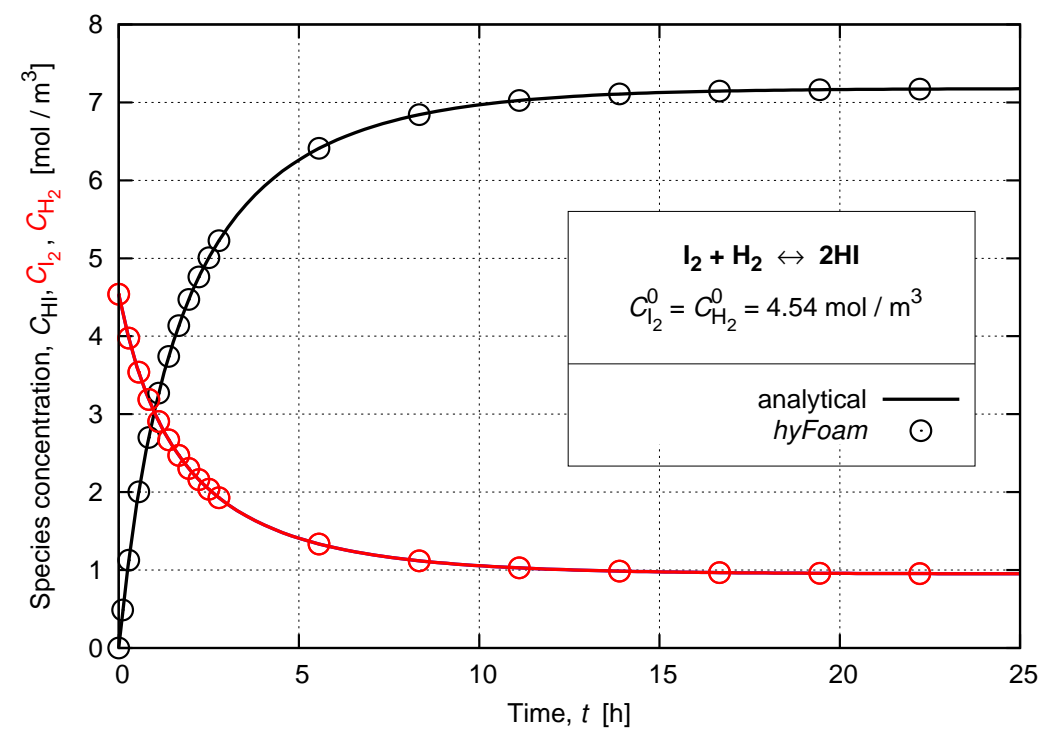

Figure 1. Species concentration versus time for a chemically reacting $\mathrm{H}_{2}-\mathrm{I}_{2}$ reservoir at a constant temperature of $700 \mathrm{~K}$ and an initial pressure of $0.528 \mathrm{~atm}$.

However, these basic chemistry features are insufficient to accurately model high-temperature dissociating gas mixtures. In order to mitigate this problem, a two-temperature approach has been adopted, making a distinct juncture between the translational-rotational and vibrational temperatures. ${ }^{22}$ 


\section{B. Governing equations}

\section{Two-temperature model}

Decomposition of the internal energy into its elementary energy modes is a consequence of the BornOppenheimer approximation. ${ }^{23}$ Although its validity has been debated by Giordano, ${ }^{24}$ this approach undoubtly remains the reference as it has proved to give satisfactory results for numerous re-entry case scenarios. ${ }^{25,26,27}$ Moreover, as Bird has indicated, when it comes to the numerical modelling of a physical phenomenon, a gain in physical realism does not necessarily translates into improved results. ${ }^{15}$

The study of the ionised flow-field is outside of the scope of this paper and as such the electron-electronic energy mode is disregarded in the following. The total energy, E, can be decomposed as the sum of the kinetic, translational, internal, and chemical energies as follows ${ }^{28}$

$$
E=\frac{1}{2} \rho u_{i} u_{i}+\sum_{k=1}^{s} E_{t, k}+\sum_{k=1}^{s} E_{r, k}+\sum_{k=1}^{s} E_{v, k}+\sum_{k=1}^{s} \rho_{k} h_{k}^{\circ}
$$

where indices $t, r$, and $v$ refer to translational, rotational, and vibrational energy modes, respectively. $k$ is an index to vary between 1 and the number of species in the mixture, $s . \rho$ is the mixture density and $u_{i}$ for $i$ to vary between 1 and 3 denotes one of three components of the velocity vector. $\rho_{k}$ stands for the partial density of species $k$ and $h_{k}^{\circ}$ is the standard enthalpy of formation of species $k$. In particular, the relation between the vibrational energy, $E_{v}$, and the vibrational energy per unit mass of species $k, e_{v, k}$, is given by

$$
E_{v}=\sum_{k=1}^{s} E_{v, k}=\sum_{k=1}^{s} \rho_{k} e_{v, k}
$$

The translational and rotational energy modes are fully excited below a few tens of Kelvin and the relaxation towards equilibrium of the translational and rotational temperatures, denoted by $T_{t}$ and $T_{r}$, respectively, is known to be achieved within a small number of particle collision events. ${ }^{15}$ Hence, $T_{t}$ and $T_{r}$ are considered to be identical, called the translational-rotational temperature, and designated by $T_{t r}$. Depending on the type of particle under investigation, the internal energies per unit mass of species may be written as

$$
\text { atom } a\left\{\begin{array} { l } 
{ e _ { t , a } = 3 / 2 \times R _ { a } T _ { t r } } \\
{ e _ { r , a } = 0 } \\
{ e _ { v , a } = 0 }
\end{array} \quad \text { (3) molecule } m \left\{\begin{array}{rl}
e_{t, m} & =3 / 2 \times R_{m} T_{t r} \\
e_{r, m} & =R_{m} T_{t r} \\
e_{v, m} & =\frac{R_{m} \theta_{v, m}}{\exp \left(\frac{\theta_{v, m}}{T_{v, m}}\right)-1}
\end{array}\right.\right.
$$

where $R$ is the specific gas constant, equal to the ratio of the universal gas constant divided by the molecular weight of the particle, and $\theta_{v}$ is the characteristic vibrational temperature of the particle of interest. A simple harmonic oscillator is employed for the vibrational mode. ${ }^{15}$

Coupling between the translational-rotational and vibrational energy modes, denoted by $Q_{k, V-T}$, is then quantified using the Landau-Teller equation. ${ }^{29}$ This equation prescribes the translational/rotationalvibrational (V-T) energy exchange rate and thus the time rate of change in vibrational energy as follows

$$
Q_{k, V-T}=\rho_{k} \frac{\partial e_{v, k}\left(T_{v, k}\right)}{\partial t}=\rho_{k} \frac{e_{v, k}\left(T_{t r}\right)-e_{v, k}\left(T_{v, k}\right)}{\tau_{k, V-T}}
$$

where $\tau_{k, V-T}$ is the molar averaged V-T relaxation time.

Millikan and White $^{30}$ proposed a semi-empirical correlation to estimate the V-T relaxation time for a large variety of species. The formula is valid for temperatures within the range [300; 8,000] K. In the worst case scenario divergence from the experimental measurements is observed up to a factor of 5 . For a mixture, this yields

$$
\tau_{k, V-T}=\tau_{k}^{M W}=\left(\sum_{j=1}^{s} X_{j} / \tau_{j k, V-T}\right)^{-1}
$$


where $X$ is the species molar fraction and the interspecies relaxation times, $\tau_{j k,{ }_{V-T}}$, can be written as

$$
\tau_{j k,,_{-T}}=\frac{1}{p} \exp \left[A_{j k}\left(T_{t r}^{-1 / 3}-0.015 \mathcal{M}_{j k}^{1 / 4}\right)-18.42\right] \text { with } p \text { in atm }
$$

Coefficient $A_{j k}$ and the reduced mass of species $j$ and $k, \mathcal{M}_{j k}$, are given by

$$
\begin{gathered}
A_{j k}=1.16 \times 10^{-3} \sqrt{\mathcal{M}_{j k}} \theta_{v, k}^{4 / 3} \\
\mathcal{M}_{j k}=\frac{\mathcal{M}_{j} \mathcal{M}_{k}}{\mathcal{M}_{j}+\mathcal{M}_{k}}
\end{gathered}
$$

where $\mathcal{M}_{k}$ is the molecular weight of species $k$ in $\mathrm{g} / \mathrm{mol}$.

Park added a correction factor to the Millikan-White formula ${ }^{31}$ to take into account the inaccurate estimation of the collision cross-section at high temperatures. The V-T relaxation time is now defined by

$$
\tau_{k, V-T}=\tau_{k}^{M W}+\tau_{k}^{P}
$$

where the collision-limited relaxation time, $\tau_{k}^{P}$, is a function of the average molecular speed, $\bar{c}_{k}$, the limited collision cross-section, $\sigma_{v}$, and the number density as follows

$$
\tau_{k}^{P}=\frac{1}{\bar{c}_{k} \sigma_{v} n_{k}}
$$

with

$$
\bar{c}_{k}=\sqrt{\frac{8 R_{k} T_{t r}}{\pi}} \quad(12) \quad \text { and } \quad \sigma_{v}=10^{-21}\left(\frac{50,000}{T_{t r}}\right)^{2}
$$

Vibrational-vibrational coupling, $Q_{V-V}$, and chemistry-vibration coupling, $Q_{V-\text { ch }}$, are beyond the scope of this paper and will be implemented and investigated in future studies.

Finally, the overall temperature, $T$, is reconstructed considering a formula involving molecular temperatures and their respective degrees of freedom. This may be written as

$$
T=\frac{3 T_{t}+\bar{\zeta}_{i n t} T_{i n t}}{3+\bar{\zeta}_{\text {int }}}
$$

An analogous expression can be found in Bird's book ${ }^{15}$ to determine the overall temperature from any DSMC computations. The subscript int refers to the internal energy modes which are in the present case the rotational and vibrational modes. $\zeta$ is the number of degrees of freedom in relation to one specific energy mode. Both translational and rotational energy modes are supposed to be fully excited, therefore, three degrees of freedom are allocated to the translational mode and $\zeta_{t}=3$. Neglecting the degree of freedom of rotation along the nuclear axis for diatomic molecules, two degrees of freedom are associated with the rotational energy mode if the particle is a molecule. Different values taken by $\zeta$ can be derived from equipartition theory, with

$$
e_{r}=\frac{1}{2} \zeta_{r} R T_{t r}
$$

and

where $\bar{\zeta}_{\text {int }}$ is the mass-weighted average of $\zeta_{r}$ and $\zeta_{v}$.

\section{Non-equilibrium Navier-Stokes-Fourier equations}

The Navier-Stokes-Fourier (NSF) equations are solved to compute transient compressible reacting flows. These are presented below in flux-divergence form for the $s$ species transport and reaction equations, the momentum equations, the vibrational energy equation, and the total energy equation. It states

$$
\frac{\partial \mathcal{U}}{\partial t}+\frac{\partial \mathcal{F}_{i}}{\partial x_{i}}=\dot{\mathcal{W}}
$$

where the vector of conserved quantities, $\mathcal{U}$, writes

$$
\mathcal{U}=\left(\rho_{1}, \rho_{2}, \cdots, \rho_{s}, \rho u, \rho v, \rho w, E_{v}, E\right)^{T}
$$


$u, v$, and $w$ are the three components of the velocity vector. The flux vectors, $\mathcal{F}_{i}$, are given by

$$
\mathcal{F}_{i}=\left(\begin{array}{c}
\rho_{1} u_{i}+\mathcal{J}_{1, i} \\
\rho_{2} u_{i}+\mathcal{J}_{2, i} \\
\vdots \\
\rho_{s} u_{i}+\mathcal{J}_{s, i} \\
\rho u_{i} u+\delta_{i 1} p+\tau_{i 1} \\
\rho u_{i} v+\delta_{i 2} p+\tau_{i 2} \\
\rho u_{i} w+\delta_{i 3} p+\tau_{i 3} \\
E_{v} u_{i}+q_{v, i}+\sum_{k=1}^{s} E_{v, k} v_{k, i} \\
\sum_{j=1}^{3} \tau_{i j} u_{j}+q_{t r, i}+q_{v, i}+\sum_{k=1}^{s} h_{k} \mathcal{J}_{k, i}
\end{array}\right)
$$

where index $i$ refers to one of the three dimensions of space and $\delta$ is the Kronecker delta. $h_{k}$ stands for the enthalpy per unit mass of species $k$. $\tau$ is the shear stress tensor and Stokes' hypothesis for the bulk viscosity is assumed to hold. Thus

$$
\tau_{i j}=-\mu\left(\frac{\partial u_{i}}{\partial x_{j}}+\frac{\partial u_{j}}{\partial x_{i}}\right)-\lambda \frac{\partial u_{k}}{\partial x_{k}} \delta_{i j} \quad \text { with } \quad \lambda=-\frac{2}{3} \mu
$$

where $\mu$ is the shear viscosity and $\lambda$ is the bulk viscosity.

Components of the heat conduction vector, $\mathbf{q}$, are assumed to follow Fourier's heat law

$$
q_{t r, i}=-\kappa_{t r} \frac{\partial T_{t r}}{\partial x_{i}} \quad q_{v, i}=-\kappa_{v} \frac{\partial T_{v}}{\partial x_{i}}
$$

where the thermal conductivity $\kappa$ is decomposed into translational-rotational and vibrational contributions. Please refer to Ref. 28 for further detail.

$\mathcal{J}_{k, i}$ are the components of the species diffusion vector for species $k$ defined as the product of the partial density $\rho_{k}$ and the corresponding component of the diffusion velocity vector, $v_{k, i}$. Their implementation in the OpenFOAM framework is planned in due course. Therefore, it can be considered in the following that diffusion velocities are equal to zero.

The source term vector, $\dot{\mathcal{W}}$, is given below

$$
\dot{\mathcal{W}}=\left(\dot{\omega}_{1}, \dot{\omega}_{2}, \cdots, \dot{\omega}_{s}, 0,0,0, \sum_{k=1}^{s}\left(Q_{k, V-T}+Q_{k, V-V}+Q_{k, V-\mathrm{ch}}\right), 0\right)^{T}
$$

where $\dot{\omega}_{k}$ is the net mass production of species $k$.

\section{Results}

\section{A. One-temperature chemistry solver validation at high temperatures}

A 0-degree adiabatic heat bath considering relaxation towards thermochemical equilibrium provides a good benchmark case to check how well the new hyFoam CFD code performs in relation to high-temperature gas chemistry. The test case is composed of a single cubic cell of length $1 \times 10^{-5} \mathrm{~m}$ and initial pressure of 0.063 atm. Five different initial temperatures have been considered to cover a range of temperatures during the molecular dissociation, these being $T^{0}=\{5,7.5,10,15,20\} \times 10^{3} \mathrm{~K}$. The time-step for CFD computations has been set at $2 \times 10^{-9} \mathrm{~s}$.

The chemical reaction considered is the irreversible molecule-molecule dissociation of oxygen

$$
\mathrm{O}_{2}+\mathrm{O}_{2} \stackrel{k_{f}(T)}{\longrightarrow} 2 \mathrm{O}+\mathrm{O}_{2}
$$

The forward rate constant, $k_{f}$, is assumed to follow the Arrhenius law, and is thus expressed by

$$
k_{f}(T)=A \times T^{\beta} \exp \left(-\frac{T_{a}}{T}\right)
$$


where $A$ is a pre-exponential factor, $\beta$ is the temperature exponent, and $T_{a}$ is the temperature of activation derived from the activation energy. The units of $A$ and $T_{a}$ are given in $\mathrm{m}^{3} / \mathrm{mol} / \mathrm{s}$ and Kelvin, respectively. Three sets of forward rate constants are employed : rates from Quantum Kinetic (QK) theory ${ }^{13}$ rates from the Dunn and Kang (DK) experiments, ${ }^{32,33}$ and Park's rates for the use of a two-temperature model (P2T). ${ }^{31}$ A single temperature has been employed to represent all of the internal modes and the rate coefficients are shown in Table 1.

Table 1. Parameters for the evalution of $k_{f}$

\begin{tabular}{cccc} 
Reaction rate & \multicolumn{4}{c}{ Arrhenius law constants } \\
& $A$ & $\beta$ & $T_{a}$ \\
\hline QK & $3.21 \times 10^{19}$ & -1.0 & $59,370.6$ \\
DK & $3.24 \times 10^{19}$ & -1.0 & $59,883.0$ \\
P2T & $2.0 \times 10^{21}$ & -1.5 & $59,500.0$ \\
\hline
\end{tabular}

In the DSMC calculations, an ensemble average of three statistically-independent solutions has been carried out. The time-step for DSMC computations has been set at $1.52 \times 10^{-9} \mathrm{~s}$ as prescribed in Ref. 13 . The comparisons between hyFoam and dsmcFoam are shown in figures 2(a) and 2(b). Results using DK rates are not displayed for reasons of clarity. P1T refers to $\mathrm{P} 2 \mathrm{~T}$ reaction rates when using a single-temperature model. Firstly, considering the QK rates for an initial temperature of 10,000 K, the hyFoam predictions are seen to be in excellent agreement with those provided by Boyd's analytical code. ${ }^{13}$ hyFoam has thus been validated for a single, irreversible chemical reaction of dissociation involving $\mathrm{O}_{2}$. However, it is evident that none of the reaction rates presented in Table 1 demonstrate a satisfactory agreement between CFD and DSMC results. For each initial temperature considered, the rate of change in temperature and species concentration is overpredicted using the CFD one-temperature model. This benchmark case illustrates the limitations of a one-temperature model for the study of dissociating mixtures. According to Bird, ${ }^{15}$ "the quantum treatment of vibration permits a physically realistic treatment of dissociation (p.52)." Therefore, it is essential to quantify the vibrational energy mode, i.e., split the internal energy into its separate energy modes, and to model the energy exchange between these modes. It is this two-temperature formulation that is discussed for single-species gases in the two subsequent paragraphs.

\section{B. Vibrational-Translational relaxation of a single-species gas}

The single-temperature OpenFOAM CFD solver hyFoam serves as the foundation of the newly coded solver hy2Foam that incorporates the two-temperature model. The 0-degree adiabatic heat bath will again provide a first benchmark case for the validation of the implementation of the two-temperature model. Emphasis is made on vibrational-translational $(\mathrm{V}-\mathrm{T})$ relaxation. For this purpose, the same single cubic cell filled with $\mathrm{O}_{2}$ is now set-up with different initial translational and vibrational temperatures. The translational temperature is set to $20,000 \mathrm{~K}$ while the vibrational temperature is lowered to $300 \mathrm{~K}$. To further simplify the problem and solely investigate V-T energy transfers, chemical reactions of dissociation are disabled. Thus, the gas is composed of a single species.

dsmcFoam intrinsically has three temperatures : translational, rotational, and vibrational. However, in the event of a rotational collision number, $Z_{r}$, set to unity, the rotational energy mode will always be in a state of thermal equilibrium and $T_{r}$ will equal $T_{t}$ to form $T_{t r}$. Tuning the value of $Z_{r}$ allows us to draw a direct comparison between the dsmcFoam and hy2Foam CFD results. Other CFD and DSMC settings remain unchanged.

Due to the inherent uncertainties arising from the experimental determination of the vibrational-translational relaxation time, $\tau_{V-T}$, it is considered unlikely that the Millikan-White correlations with (MWP) or without (MW) Park's correction will match DSMC results. Therefore, an attempt is made to derive the time evolution of $\tau_{V-T}$ from DSMC simulations to later decide on the validity of hy2Foam implementation. Equation 5 is employed and working backwards : knowing the $T_{t r}(t)$ and $T_{v}(t)$ profiles, the vibrational energy and its time derivative are computed. This yields in a system of a single equation with one unknown whose result is depicted with crosses shown in Figure 3. A curve-fitted profile denoted by the solid line is drawn and is then injected into CFD calculations. As expected, it can be seen that MW correlations clearly underpredict the value of $\tau_{V-T}$. 


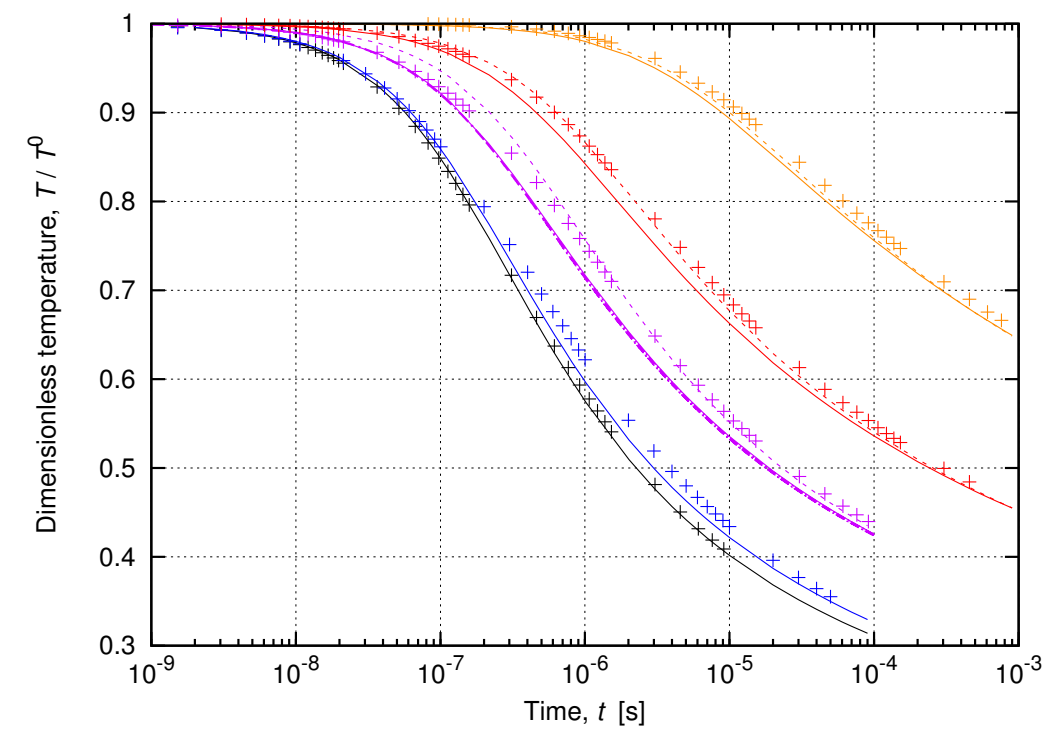

(a) Gas mixture overall temperature distribution versus time

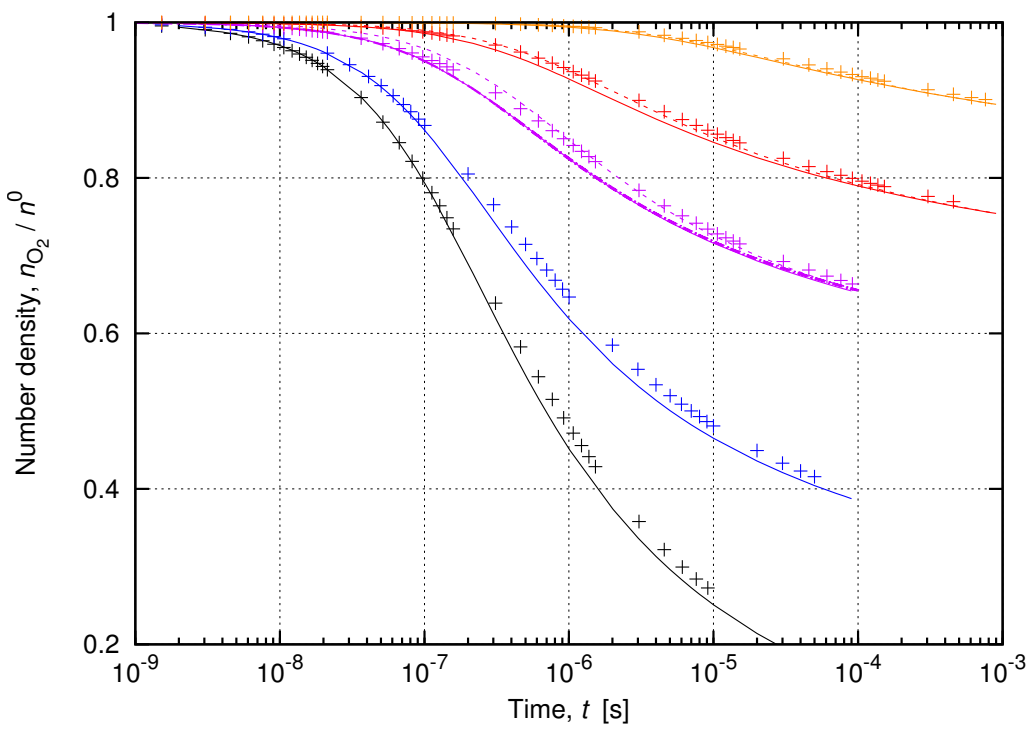

(b) $\mathrm{O}_{2}$ molecule concentration versus time

Figure 2. Dissociation of an $\mathrm{O}_{2}$ reservoir from a given initial temperature and a pressure of 0.063 atm. The crosses are the ensemble averages resulting from DSMC computations. The solid and dashed lines display the results using hyFoam CFD solver along with QK and P1T rates, respectively. The violet dashed-dotted line is the distribution resulting from the use of the analytical code of Boyd. Initial temperatures $T^{0}=$ $\{5,7.5,10,15,20\} \times 10^{3} \mathrm{~K}$ are associated with colors yellow, red, violet, dark blue, and black, respectively.

This results in vibrational relaxation to equilibrium at an excessive pace. Adding Park's correction for high temperature mixtures does contribute to significantly increasing the V-T relaxation time but it does not translate into a better concordance with DSMC data for this set of initial conditions. A significant departure from the DSMC data is observed for $t$ no greater than $3 \times 10^{-7} \mathrm{~s}$ so that a slower thermal relaxation is also evident for quantities such as the molecular temperatures and pressure as shown in Figure 4. These discrepancies are easily understood by the fact that temperatures are outside of the temperature range suitable for the MW correlation and by the very strong degree of thermal non-equilibrium that this case scenario presents.

In Figure 4, both molecular temperatures and pressure profiles are in excellent concurrence with DSMC results. MW and MWP simulations achieve a state of thermal equilibrium 2.5 times faster and 1.08 times 


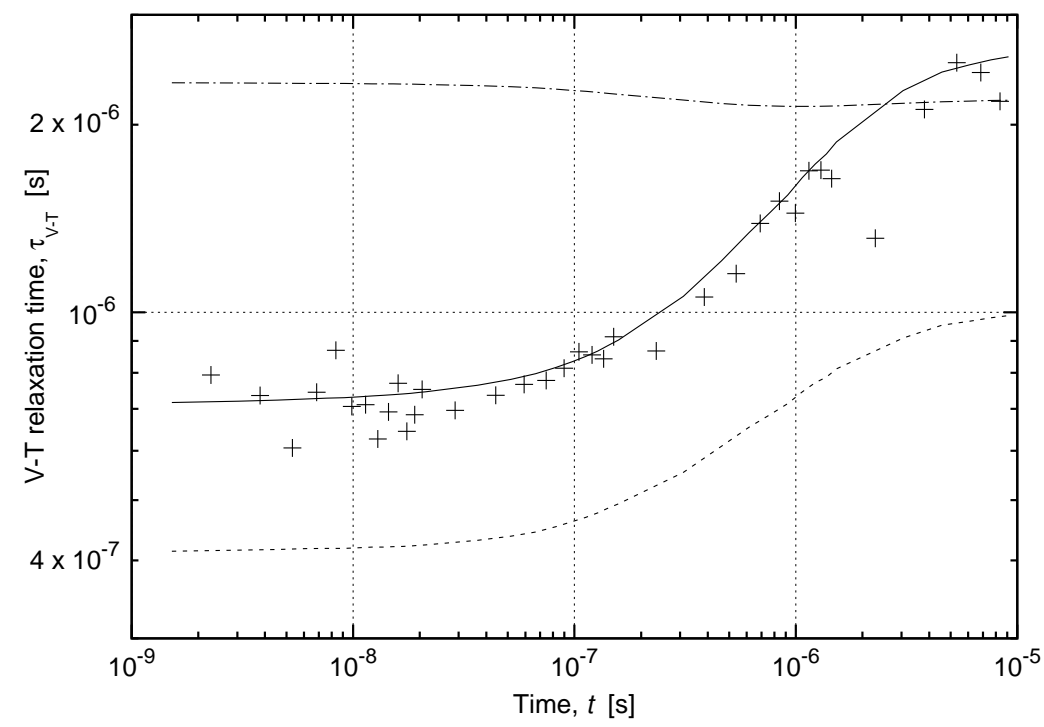

Figure 3. V-T relaxation time parameter versus simulation time for an $\mathrm{O}_{2}$ reservoir from an initial translational-rotational temperature of $20,000 \mathrm{~K}$, vibrational temperature of $300 \mathrm{~K}$, and a pressure of 0.063 atm. The crosses are ensemble averages resulting from the use of DSMC data to recover $\tau_{V-T}$. The solid line represents the curve-fitted profile. The dashed line and dashed-dotted line display the results using MW and MWP correlations, respectively.

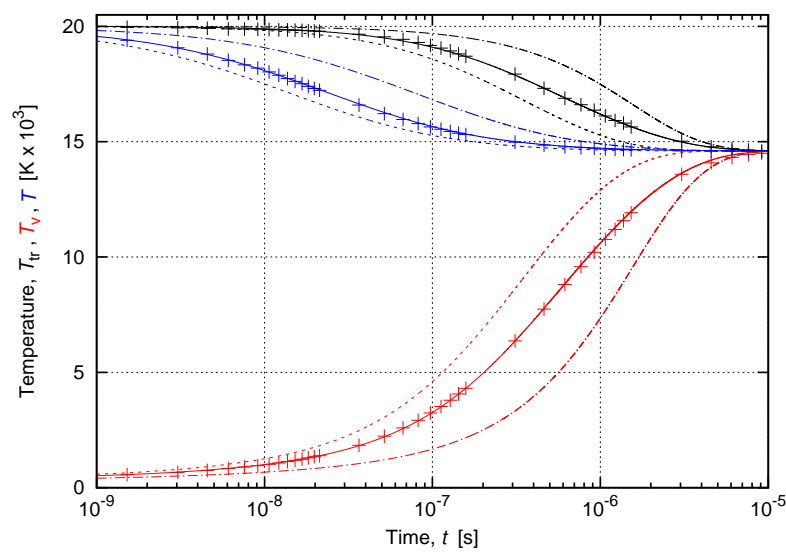

(a) Translational (black), vibrational (red), and overall (blue) temperatures distributions versus time

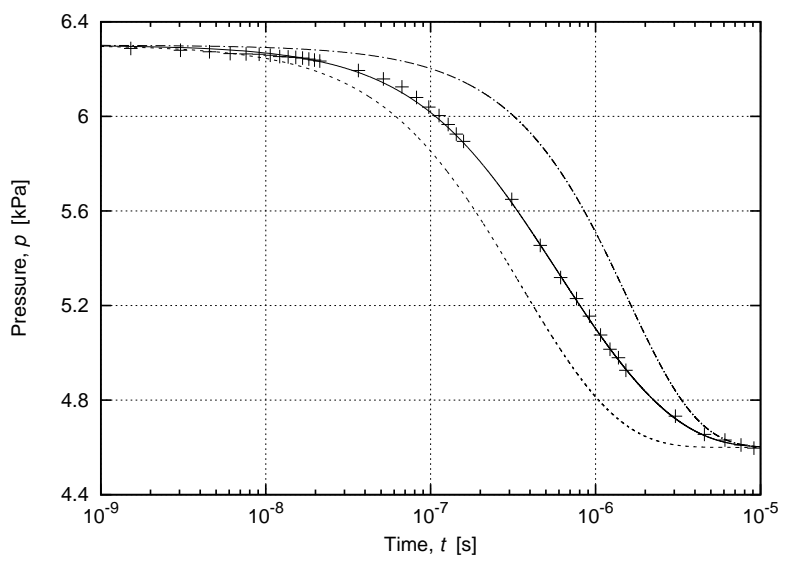

(b) Pressure distribution versus time

Figure 4. V-T relaxation of an $\mathrm{O}_{2}$ reservoir from an initial translational-rotational temperature of $20,000 \mathrm{~K}$, vibrational temperature of $300 \mathrm{~K}$, and a pressure of $0.063 \mathrm{~atm}$. The crosses are ensemble averages resulting from DSMC computations. Any solid lines represent hy2Foam results using DSMC's $\tau_{V-T}$ curve-fitted profile. Any dashed lines and dashed-dotted lines display the results using MW and MWP correlations, respectively.

slower, respectively (defined as the time to recover $99 \%$ of the equilibrium temperature). The initial disturbed system restores to a state of equilibrium at a temperature of $14,600 \mathrm{~K}$. The equilibrium temperature recovered is identical for CFD and DSMC. Following the formulae provided in Ref. 15, the number of vibrational degrees of freedom associated with the simple harmonic model of vibration in oxygen at $14,600 \mathrm{~K}$ is $\zeta_{v}=1.849$. The total number of degrees of freedom is thus equal to $\zeta_{t}+\zeta_{r}+\zeta_{v}=6.849$. A straightforward calculation from the value taken by the equilibrium temperature then allows us to determine that the initial energy pool was set at a temperature of $14,600 \times 6.849 / 5=19,999 \mathrm{~K}$. Results are thus consistent with energy equipartition. 
Other case scenarios have been tested by varying the initial translational-rotational temperature for $\mathrm{O}_{2}$ (Figure 5(a)) or changing the species to be $\mathrm{N}_{2}$ or NO (Figure 5(b)). The initial number density was held constant and equal to $n^{0}=2.282 \times 10^{22} \mathrm{~m}^{-3}$. Conclusions similar to the previous paragraph can be drawn regarding the use of MW correlation and DSMC results to recover $\tau_{V-T}$. As for the MWP correlation, the thermal relaxation becomes faster for $T_{t r}^{0}$ no greater than $15,000 \mathrm{~K}$ and the specific case of the $\mathrm{N}_{2}$ molecule. More generally MWP is to be prefered to MW for each of these case scenarios.

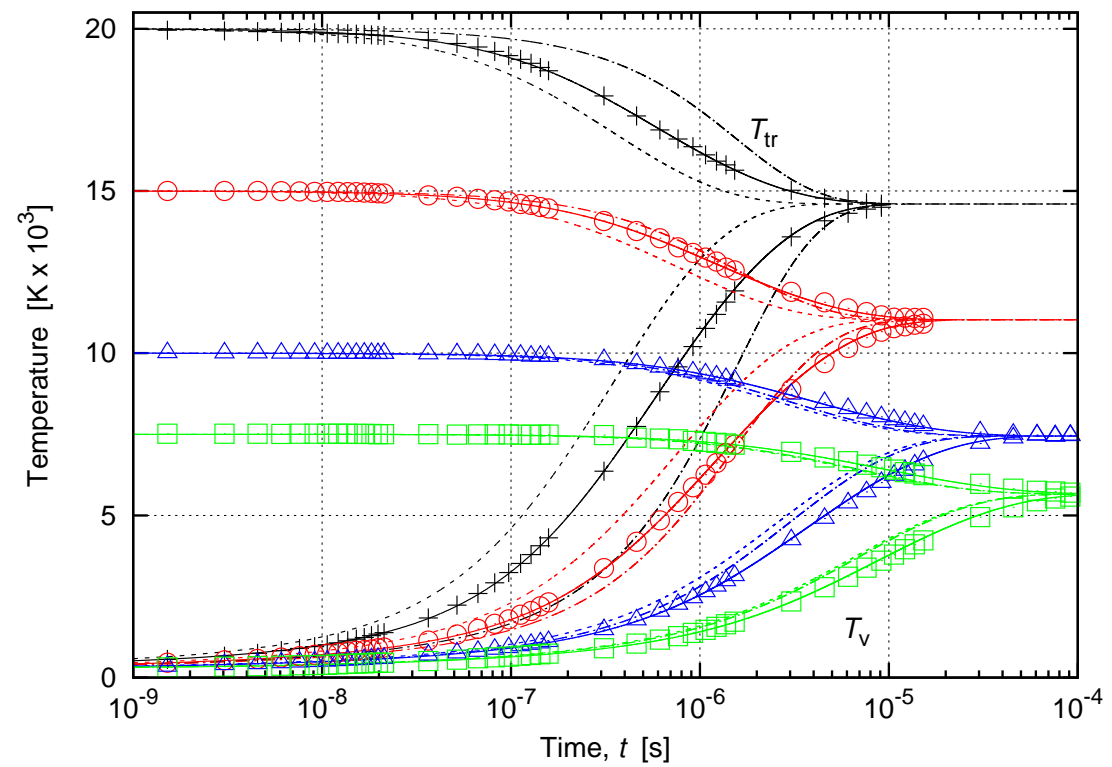

(a) Molecular temperatures distributions versus time for different $T_{t r}^{0}$

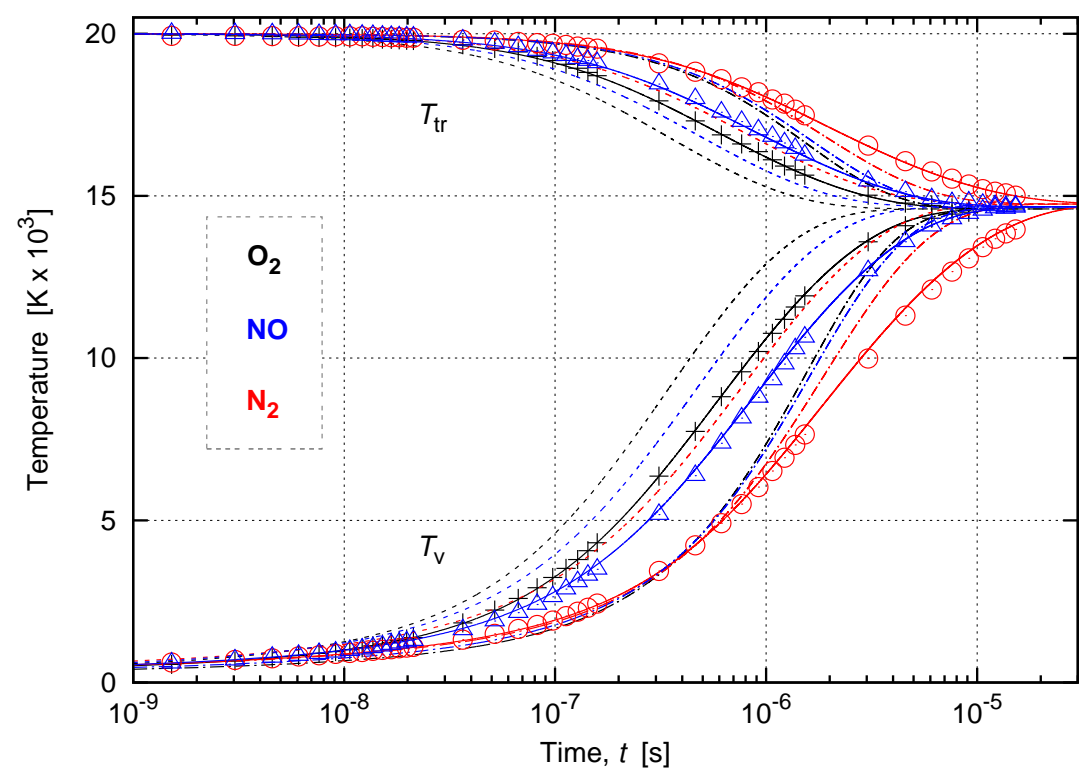

(b) Molecular temperatures distributions versus time for different molecules

Figure 5. V-T relaxation of a heat bath considering several initial translational-rotational temperatures and each of the three molecules composing a five-species air mixture. The symbols are ensemble averages resulting from DSMC computations. Any solid lines represent hy2Foam results using DSMC's $\tau_{V-T}$ curve-fitted profile. Any dashed lines and dashed-dotted lines display the results using MW and MWP correlations, respectively.

Therefore, hy2Foam results shown in Figures 4 and 5 validate the implementation of the two-temperature CFD model for a single-species gas. 


\section{Discussion on the V-T relaxation time}

In DSMC computations, the rotational collision number was set to unity to enable a direct comparison between the DSMC and CFD results. Thus, the translational and rotational energy pools were considered to be in thermal equilibrium after a single particle collision event. While this restriction has shown to be very useful to validate the implementation of hy2Foam, a typical value of 5 is usually adopted. ${ }^{15}$ In this latter case, the V-T relaxation time will differ from the case $Z_{r}=1$ due to rotational-translational and rotational-vibrational energy transfers.

Since no current correlation model can satisfactorily predict case scenarios that depart significantly from weak non-equilibrium conditions, it is thought that the DSMC method could mitigate this gap. Indeed, the determination of the V-T relaxation time from DSMC computations seems to be promising. It has yielded to a very satisfactory agreement for a given set of initial conditions. The question is whether a generalisation to any degree of thermal non-equilibrium is possible.

A preliminary formulation is proposed in Equation 24 for a single-species gas. It takes the form of the Millikan-White correlation, corrected by Park, and introduces a series of coefficients $\Lambda$ to tune accordingly. The vibrational temperature now enters into the calculation of the V-T relaxation time and aims at modelling cases with various degrees of non-equilibrium.

$$
p \times \tau_{i, V-T}=\exp \left[\theta_{v, i}^{4 / 3} \sqrt{\frac{\mathcal{M}_{i}}{2}} \Lambda_{a}\left(T_{t r}^{\Lambda_{b}}+\Lambda_{c}\left(\frac{\mathcal{M}_{i}}{2}\right)^{1 / 4}+\Lambda_{d}\left(\frac{T_{v, i}}{\theta_{v, i}}\right)^{\Lambda_{e}}\right)+\Lambda_{f}\right]+\frac{\Lambda_{g}}{\bar{c}_{i} \sigma_{v} n_{i}}
$$

Following Equation 24, Table 2 shows values of the various parameters used to produce the results shown in Figures 3, 4, and 5. Case scenarios 1-4 are reported in Figure 6(a). Kim and Boyd ${ }^{34}$ have performed a master equation analysis for $\mathrm{N}+\mathrm{N}_{2}$ considering an adiabatic heat bath in strong and weak non-equilibrium conditions. It can be observed that the different profiles of $p \times \tau_{V-T}$ versus $T_{t r}^{-1 / 3}$ exhibit a similar trend to those presented in the aforementioned paper. The slope of DSMC best fit (solid line) strongly diverges from both MW and MWP correlations for the greatest $T_{t r}^{0}$ considered. This slope does not appear to alter significantly as $T_{t r}^{0}$ decreases. As observed in Table 2, the values of the coefficients $\Lambda_{d}$ and $\Lambda_{e}$ that are responsible for these non-linear profiles appear to be constant for values of $T_{t r}^{0}$ no greater than $15,000 \mathrm{~K}$ while recalling again that $T_{v}^{0}$ is fixed here to $300 \mathrm{~K}$. Finally, $\tau_{V-T}$ is not appreciably affected by the change in $Z_{r}$ for the case scenarios that have been investigated.

Table 2. Coefficients $\Lambda$ for the evaluation of $\tau_{i,{ }_{V-T}}$ in the case $Z_{r}=1$

\begin{tabular}{|c|c|c|c|c|c|c|c|c|c|c|}
\hline \multicolumn{4}{|c|}{ Case scenarios } & \multicolumn{7}{|c|}{ Coefficients } \\
\hline no & species & $T_{t r}^{0}[\mathrm{~K}]$ & $T_{v}^{0}[\mathrm{~K}]$ & $\Lambda_{a} \times 10^{-3}$ & $\Lambda_{b}$ & $\Lambda_{c} \times 10^{-2}$ & $\Lambda_{d} \times 10^{-3}$ & $\Lambda_{e}$ & $\Lambda_{f}$ & $\Lambda_{g}$ \\
\hline & $\mathrm{O}_{2}$ & any & IW & 1.16 & $-1 / 3$ & -1.5 & 0 & 0 & -18.42 & 0 \\
\hline & $\mathrm{O}_{2}$ & any 1 & WP & 1.16 & $-1 / 3$ & -1.5 & 0 & 0 & -18.42 & 1 \\
\hline 1 & $\mathrm{O}_{2}$ & 20,000 & 300 & 1.16 & $-1 / 3$ & -1.5 & 1.0 & 1.0 & -18.42 & 0.15 \\
\hline 2 & $\mathrm{O}_{2}$ & 15,000 & 300 & 1.16 & $-1 / 3$ & -1.46 & 1.5 & 0.95 & -18.42 & 0.18 \\
\hline 3 & $\mathrm{O}_{2}$ & 10,000 & 300 & 1.16 & $-1 / 3$ & -1.45 & 1.5 & 0.95 & -18.42 & 0.18 \\
\hline 4 & $\mathrm{O}_{2}$ & 7,500 & 300 & 1.16 & $-1 / 3$ & -1.45 & 1.5 & 0.95 & -18.42 & 0.18 \\
\hline 5 & $\mathrm{~N}_{2}$ & 20,000 & 300 & 1.16 & $-1 / 3$ & -1.5 & 1.4 & 1.0 & -18.42 & 0.84 \\
\hline 6 & $\mathrm{NO}$ & 20,000 & 300 & 1.16 & $-1 / 3$ & -1.47 & 1.1 & 0.9 & -18.42 & 0.17 \\
\hline
\end{tabular}

In Figure 6(b), the influence of $T_{v}^{0}$ is highlighted for $T_{t r}^{0}$ fixed at $20,000 \mathrm{~K}$. It is interesting to note that while MWP is normally employed in high-temperature conditions, MW performs better as $T_{v}^{0}$ approaches $T_{t r}^{0}$. The coefficient $\Lambda_{g}$ has been introduced for this purpose. For weak non-equilibrium conditions (here $T_{v}^{0}$ $=18,000 \mathrm{~K}$ ), the results demonstrate excessive scatter which makes curve-fitted profiles difficult to produce. However, the linear response formulated by Millikan-White seems valid even at such temperatures that are clearly out of the temperature validity range. 


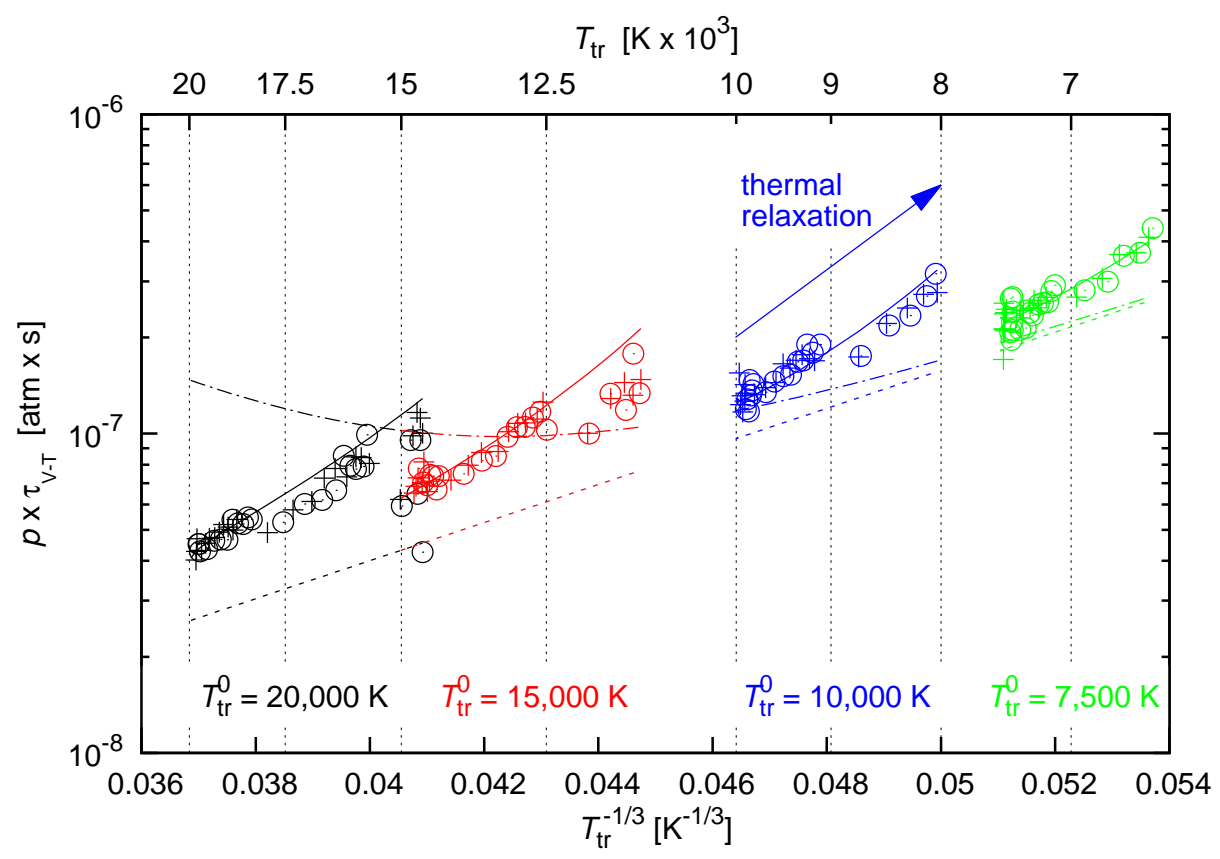

(a) Constant $T_{v}^{0}$ of $300 \mathrm{~K}$ (case scenarios 1-4 in Table 2)

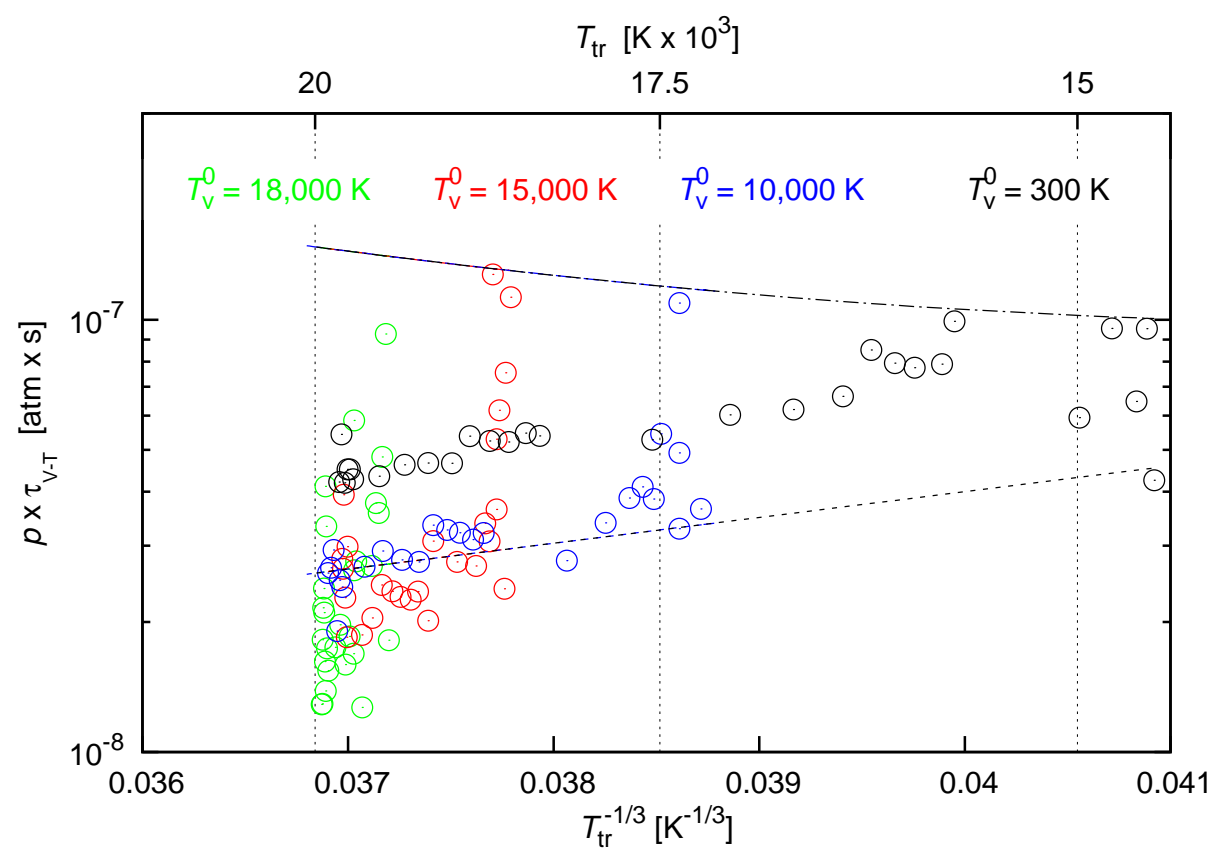

(b) Constant $T_{t r}^{0}$ of $20,000 \mathrm{~K}$

Figure 6. V-T relaxation time versus $T_{t r}^{-1 / 3}$ for an $\mathrm{O}_{2}$ adiabatic heat bath with a constant initial number density of $2.282 \times 10^{22} \mathrm{~m}^{-3}$. The crosses and circles are the ensemble averages resulting from the use of DSMC data to recover $\tau_{V-T}$ with $Z_{r}=1$ and $Z_{r}=5$, respectively. The solid line represents the curve-fitted profile. The dashed line and dashed-dotted line display the results using MW and MWP correlations, respectively.

It is obvious that a greater diversity of DSMC simulations should be run in order to ascertain noticeable patterns in the evolution of the $\Lambda$ coefficients. Future work will therefore consist of enlarging the DSMC data set and running a least-squares algorithm to obtain a best-fit. It should be further pointed out that only heating case scenarios $\left(T_{v}^{0}<T_{t r}^{0}\right)$ have been so far considered and their cooling counterparts should be investigated as well. 


\section{Conclusion}

A new open-source CFD solver, hyFoam, has been implemented into the OpenFOAM framework for the prediction of hypersonic reacting flows. The solver has first been validated for a 0 -D adiabatic heat bath considering the reversible reaction of hydrogen and iodine $\mathrm{I}_{2}+\mathrm{H}_{2} \rightleftharpoons 2 \mathrm{HI}$. This work has then been extended to high-temperature conditions with the study of the dissociation of oxygen for several initial temperatures. In comparison with a previously published analytical code, hyFoam is shown to perform equally well. Considering the dissociation reaction of oxygen, the CFD and DSMC results were in satisfactory agreement considering the limitations in the use of a single-temperature CFD model. Efforts have been undertaken to implement the translational/rotational-vibrational energy exchange rate in a two-temperature configuration, to produce a new solver called hy2Foam. hy2Foam has been validated for a 0-D adiabatic heat bath consisting of single-species gases initially set in a state of thermal non-equilibrium.

Future work will consider the generalisation of the two-temperature model to multi-species mixtures and case scenarios with a spatial dimension. A wider range of chemical reactions will be assessed including molecule-molecule and molecule-atom dissociation reactions in a 5-species air mixture composed of $\mathrm{O}_{2}, \mathrm{O}$, $\mathrm{N}_{2}$, N, and NO. ${ }^{13}$ Further studies will also be carried out in order to assess how the DSMC simulations could potentially provide a corrected formulation to the current state-of-the-art CFD V-T relaxation time semi-empirical correlations. This study is the premise to a future open-source hybrid CFD-DSMC solver. In this respect, the work carried out on improving the capabilities of the CFD solver is also important in order to achieve a satisfactory compatibility between the two distinct numerical approaches.

\section{References}

${ }^{1}$ Brown, R. E., "The future of air travel: Dinner in Sydney, London in time for 'The X-Factor'?" The Washington Post, Oct. 2014.

${ }^{2}$ Salas, M. D., "A Review of Hypersonics Aerodynamics, Aerothermodynamics and Plasmadynamics Activities within NASA's Fundamental Aeronautics Program," Aiaa 2007-4264, 2007.

${ }^{3}$ Feldick, A. M., Modest, M. F., Levin, D. A., Gnoffo, P., and Johnston, C. O., "Examination of Coupled Continuum Fluid Dynamics and Radiation in Hypersonic Simulations," AIAA Aerospace Sciences Meeting, 2009.

${ }^{4}$ Candler, G. V., Write, M. J., and McDonald, J. D., "Data-Parallel Lower-Upper Relaxation Method for Reacting Flows," AIAA Journal, Vol. 32, No. 12, 1994, pp. 2380-2386.

${ }^{5}$ Cheatwood, F. M. and Gnoffo, P. A., "User's Manual for the Langley Aerothermodynamic Upwind Algorithm (LAURA)," Tech. rep., 1996.

6 "VULCAN-CFD official website," http://vulcan-cfd.larc.nasa.gov/, Accessed: 22 May 2015.

${ }^{7}$ Nompelis, I., Drayna, T. W., and Candler, G. V., "Development of a Hybrid Unstructued Implicit Solver for the Simulation of Reacting Flows Over Complex Geometries," Aiaa paper 2004-2227, 2004.

${ }^{8}$ Nompelis, I., Drayna, T. W., and Candler, G. V., "A Parallel Unstructured Implicit Solver for Hypersonic Reacting Flow Simulation," Aiaa paper 2005-4867, 2005.

${ }^{9}$ Scalabrin, L. C. and Boyd, I. D., "Development of an Unstructured Navier-Stokes Solver For Hypersonic Nonequilibrium Aerothermodynamics," Aiaa paper 2005-5203, June 2005.

${ }^{10}$ Scalabrin, L. C. and Boyd, I. D., "Numerical Simulation of Weakly Ionized Hypersonic Flow for Reentry Configurations," Aiaa paper 2006-3773, June 2006.

${ }^{11}$ Park, C., "On Convergence of Computation of Chemically Reacting Flows," Progress in Astronautics and Aeronautics, Vol. 103, 1986, pp. 478-513.

12 "OpenFOAM official website," http://www.openfoam.com/, Accessed: 22 May 2015.

${ }^{13}$ Scanlon, T. J. et al., "Open souce Direct Simulation Monte Carlo (DSMC) chemistry modelling for hypersonic flows," AIAA Journal, Vol. 53, No. 6, 2015, pp. 1670-1680.

${ }^{14}$ Cassineli Palharini, R., Atmospheric Reentry Modelling Using an Open-Source DSMC Code, Ph.D. thesis, University of Strathclyde, Glasgow, 2014.

${ }^{15}$ Bird, G. A., The DSMC Method, Sydney, 2nd ed., 2013, ISBN : 9781492112907.

${ }^{16}$ Borgnakke, C. and Larsen, P. S., "Statistical collision model for simulating polyatomic gas with restrited energy exchange," Rarefied Gas Dynamics, Vol. 1, 1974, Paper A7, DFVLR Press, Porz-Wahn, Germany.

${ }^{17}$ Greenshields, C. J., Weller, H. G., Gasparini, L., and Reese, J. M., "Implementation of semi-discrete, non-staggered central schemes in a collocated, polyhedral, finite volume framework, for high-speed viscous flows," International Journal For Numerical Methods In Fluids, Vol. 63, No. 1, 2010, pp. 1-21.

${ }^{18}$ Kurganov, A., Noelle, S., and Petrova, G., "Semi-discrete central-upwind schemes for hypersonic conservation laws and Hamilton-Jacobi equations," SIAM J. Sci. Comput., Vol. 23, No. 3, 2001, pp. 707-740.

${ }^{19}$ Vos, J. B., Rizzi, A., Darracq, D., and Hirschel, E. H., "Navier-Stokes solvers in European aircraft design," Progress in Aerospace Sciences, Vol. 38, 2002, pp. 601-697.

${ }^{20}$ Scanlon, T. J., Cassineli Palharini, R., White, C., Espinoza, D., and Casseau, V., "Simulations of rarefied and continuum hypersonic flow over re-entry objects," Paper presented at 8th European Symposium on Aerothermodynamics for Space Vehicles, Lisbon, Portugal, March 2015. 
${ }^{21}$ Williams, F. A., Combustion Theory, Addison-Wesley, Redwood City, CA, 2nd ed., 1985.

${ }^{22}$ Park, C., "Assessment of a Two-Temperature Kinetic Model for Dissociating and Weakly Ionizing Nitrogen," Journal of Thermophysics and Heat Transfer, Vol. 2, No. 1, 1988, pp. 8-16.

${ }^{23}$ Born, R. and Oppenheimer, M., "Zur Quantentheorie der Moleküle," Annalen der Physik, Vol. 84, 1941, pp. 457-484.

${ }^{24}$ Giordano, D., "Impact of the Born-Oppenheimer approximation on aerothermodynamics," Journal of Thermophysics and Heat Transfer, Vol. 21, No. 3, 2007, pp. 647-657.

${ }^{25}$ Park, C., Howe, J. T., Jaffe, R. L., and Candler, G. V., "Review of Chemical-Kinetic Problems of Future NASA Missions, II: Mars Entries," Journal of Thermophysics and Heat Transfer, Vol. 8, No. 1, 1994, pp. 9-23.

${ }^{26}$ Olynick, D., Taylor, J. C., and Hassan, H. A., "Comparisons Between Monte Carlo Methods and Navier-Stokes Equations for Re-Entry Flows," Journal of Thermophysics and Heat Transfer, Vol. 8, No. 2, 1994, pp. 251-258.

${ }^{27}$ Hash, D. et al., "FIRE II Calculations for Hypersonic Nonequilibrium Aerothermodynamics Code Verification: DPLR, LAURA, US3D, and LeMANS," Aiaa paper 2007-605, 2007.

${ }^{28}$ Candler, G. V. and Nompelis, I., "Computational Fluid Dynamics for Atmospheric Entry," Rto-en-avt-162, 2009.

${ }^{29}$ Laudau, L. and Teller, E., "Theory of sound dispersion," Physikalische Zeitschrift der Sowjetunion, Vol. 10, 1937.

${ }^{30}$ Millikan, R. C. and White, D. R., "Systematics of Vibrational Relaxation," The Journal of Chemical Physics, Vol. 39, No. 12, 1963, pp. 3209-3213.

${ }^{31}$ Park, C., Nonequilibrium Hypersonic Aerothermodynamics, Wiley International, New York, 1990.

${ }^{32}$ Dunn, M. G. and Kang, S. W., "Theoretical and Experimental Studies of Reentry Plasmas," Nasa cr-2232, April 1973.

${ }^{33}$ Bussing, T. and Eberhardt, S., "Chemistry Associated with Hypersonic Vehicles," Aiaa paper 87-1292, 1987.

${ }^{34}$ Kim, J. G. and Boyd, I. D., "Master Equation Analysis of Thermochemical Nonequilibrium of Nitrogen," Aiaa paper 2012-3305, 2012. 